\title{
Dual fluid for the Kerr black hole
}

\section{Vyacheslav Lysov}

Walter Burke Institute for Theoretical Physics, California Institute of Technology, Pasadena, CA 91125, U.S.A.

E-mail: lysov.slava@gmail.com

ABSTRACT: Rotating black holes are algebraically special solutions to the vacuum Einstein equation. Using properties of the algebraically special solutions we construct the dual fluid, which flows on black hole horizon. An explicit form of the Kerr solution allows us to write an explicit dual fluid solution and investigate its stability using energy balance equation. We show that the dual fluid is stable because of high algebraic speciality of the Kerr solution.

Keywords: Black Holes, Classical Theories of Gravity, Holography and condensed matter physics (AdS/CMT)

ARXIV EPRINT: 1712.08079 


\section{Contents}

1 Introduction 1

2 The fluid/gravity correspondence $\quad 3$

3 Kerr geometry 4

$3.1 r=r_{0}=$ const surface $\quad 5$

3.2 Induced metric 5

$\begin{array}{lll}3.3 & \text { Extrinsic curvature } & 6\end{array}$

3.4 Null frame $\quad 6$

4 Slowly rotating black hole $\quad 7$

$\begin{array}{lll}4.1 & \text { Geometry } & 7\end{array}$

4.2 Dual fluid $\quad 9$

$\begin{array}{lll}4.3 & \text { Type } \mathbf{I}_{i} \text { fluid as a Killing flow } & 10\end{array}$

5 Dual fluid for rotating black hole $\quad 11$

5.1 Geometry 11

$\begin{array}{lll}5.2 & \text { Near-horizon expansion } & 12\end{array}$

$\begin{array}{lll}5.3 & \text { The type } \mathbf{I}_{i} \text { fluid as a Killing flow } & 13\end{array}$

6 Stability, Reynolds number, turbulence $\quad 14$

$\begin{array}{lll}7 & \text { Summary of the results } & 16\end{array}$

\section{Introduction}

The fluid/gravity correspondence is a framework that connects the knowledge about the gravity equations and fluid dynamics. There are multiple approaches to such correspondence [1-9] that are mostly inspired by the membrane paradigm and AdS/CFT. Given a fluid/gravity mapping we can naturally ask two major questions:

1. Given known solution to nonlinear equations on either fluid or gravity side, can we generate new solution to dual nonlinear equation via duality map?

2. What is the relation between stability properties of the dual solutions?

The first question is interesting as it is typically very hard to solve nonlinear equations, so duality map that generates new solutions from known solutions is very useful. The second question is interesting because the fluid stability is quite well studied as it closely related to the turbulence phenomenon. Typically the transition between the stable and unstable 
regimes is controlled by a single dimensionless Reynolds number, so it is reasonable to search for a corresponding gravity dual.

Among the multiple fluid/gravity mappings the algebraic speciality approach fits best to answer questions that we described. In simple terms our fluid/gravity map is very similar to membrane paradigm with algebraic speciality constraints providing additional equations to make the number of variables and equations identical. Similarly, AdS-based perturbative solutions are algebraically special at leading order. In our paper [9] we described the simplest case of the fluid/gravity map with minimally special geometry and (conformally) flat fluid hypersurface geometry. Unfortunately almost all known solutions to Einstein equations are more special [10], i.e. have additional vanishing Weyl tensor components. Furthermore, the horizon geometry is far from being (conformally) flat for most solutions. Thus, we need to modify our approach to adopt for the known black hole solutions. While it is possible to describe the most general algebraic constrains and hypersurface geometry, we decided to restrict our discussion to the rotating black holes. Such restriction allows us to make discussion much simpler while keeping several interesting phenomena and being physically relevant on both gravity and fluid sides.

The generic rotating black hole geometry turns out to be very complicated to analyze straight on, so we start our discussion with slowly rotation case, where the angular momentum $J$ is much smaller then the mass squared $M^{2}$. The geometry is an algebraic type $\mathbf{D}$, while the fluid hypersurface preserves the smaller type $\mathbf{I}_{i}$ subset of type $\mathbf{D}$ constraints. The type $\mathbf{I}_{i}$ includes type $\mathbf{I}$ constraints, so we can construct the dual fluid, while the additional constraints restrict possible fluid solutions to Killing flows. Interestingly, the simple analysis of additional speciality constraints provides a quick way solve the Navier-Stokes (NS) equations and to reproduce the slowly rotating black hole results from [11]. Since slowly rotating Kerr solution can be viewed as a small perturbation over the Schwarzschild geometry our NS system and fluid solution match with the results by Bredberg and Strominger [12].

The fluid dual to the generic rotating black hole solution obeys the modified version of an incompressible NS equation. The additional terms are similar to ones described in fluid/gravity generalizations [13]. Similarly to the toy case higher algebraic speciality provides additional constraints on velocity similar to the Killing equation. A solution to additional constraints provides a quick way of solving the NS equation for a dual fluid. As an example we describe in details both fluid equations and solution for the case of $4 \mathrm{~d}$ Kerr geometry.

Given an explicit form of the fluid equations and solutions we can investigate the linearized stability of the flow. The most common approach to stability is based on the Reynolds number estimation. The Reynolds number for the slow rotating case is equal to the $3 J / M^{2}$ and is parametrically small. We also estimate the critical Reynolds number, where fluid can be unstable, using the energy balance equation. It turns out that the dual fluid to the generic type $\mathbf{D}$ black hole solution is always stable.

We begin our discussion with the review of the algebraically special fluid/gravity correspondence in section 2. In section 3 we review the Kerr geometry and describe the dual fluid hypersurface. In section 4 we consider a slow rotating Kerr solution and apply the fluid/gravity map to it. In section 5 we describe the dual fluid to the Kerr black hole and 
discuss its stability in section 6 . In the closing section 7 we discuss the results and possible generalizations.

\section{The fluid/gravity correspondence}

In this section we will review the fluid/gravity correspondence and modify it to include the rotating black hole solutions.

Given a solution to the Einstein equations in $p+2$ dimensions and a time-like codimension-one hypersurface $\Sigma$ we can construct a symmetric conserved two-tensor $T_{a b}$

$$
\nabla^{a} T_{a b}=0, \quad T_{a b}=T_{b a}, \quad a, b=0, \ldots, p,
$$

where $\nabla^{a}$ is a covariant derivative with respect to the induced metric $h_{a b}$ on a hypersurface. The tensor $T_{a b}$ is often called the Brown-York (BY) tensor and is constructed from the extrinsic curvature

$$
T_{a b} \equiv K_{a b}-\frac{1}{2} h_{a b} K, \quad K_{a b}=\frac{1}{2} \mathcal{L}_{n} h_{a b},
$$

with $n$ being the unit normal to $\Sigma$ and $K \equiv K_{a b} h^{a b}$. The fluid/gravity correspondence maps the BY tensor into the fluid stress tensor. Fluid equations can be formulated in the form of covariant conservation for the fluid stress tensor. However the $(p+1)(p+2) / 2$ components of the stress tensor are expressed in terms of $p$-components of the fluid velocity and two scalars: density and pressure. In case of gravity-dual fluid the additional constraints for the BY tensor come from metric being algebraically special.

The covariant conservation (2.1) originates from the bulk Einstein equation $G_{\mu \nu}=0$ $\mu, \nu=0, \ldots, p+1$ on the hypersurface $\Sigma$ with coordinates $x^{a}, a=0, \ldots, p$

$$
0=\left.G_{\mu b} n^{\mu}\right|_{\Sigma}=\nabla^{a} T_{a b}=\nabla^{a} K_{a b}-\partial_{b} K
$$

and

$$
0=\left.2 G_{\mu \nu} n^{\mu} n^{\nu}\right|_{\Sigma}=K^{2}-K_{a b} K^{a b}-R^{(p+1)},
$$

where we use $(p+1)$ upperscript for the hypersurface quantities. The minimally special metrics are algebraic type $\mathbf{I}$, what imposes a constraint

$$
C_{\mathrm{lilj} j} \equiv C_{\mu \nu \lambda \rho} \mathbf{l}^{\mu} m_{i}^{\nu} \mathbf{l}^{\lambda} m_{j}^{\rho}=0, \quad i, j=1, \ldots, p,
$$

where $C_{\mu \nu \lambda \rho}$ is the Weyl tensor and $\mathbf{l}, \mathbf{k}, m_{i}$ are the null frame vectors. The number of additional constraints in (2.5) is just enough to make equal the number of BY tensor components and the total number of fluid equations (2.3), (2.4).

We often have a solution to Einstein equation and a null frame where solution is maximally special. The rotational black holes are type $\mathbf{D}$ solutions in four dimensions and type $\mathbf{I I}_{i}$ in higher dimensions. Type $\mathbf{I I}_{i}$ contains type $\mathbf{I}$ as a subset, so we have freedom of choosing the hypersurface $\Sigma$ and its time-foliation to preserve some subset of algebraic constraints. In particular we want the null frame vectors to be

$$
\sqrt{2} \mathbf{l}=T-n, \quad \sqrt{2} \mathbf{k}=T+n,
$$


with $T$ being unit normal for the time foliation $\Sigma_{T}$ of $\Sigma$. The rest of the null frame vectors $m_{i}$ are orthonormal basis for tangent space to $\Sigma_{T}$.

We can express the $p+2$-dimensional Riemann tensor on a hypersurface in terms of intrinsic geometry and extrinsic curvature $K_{a b}$

$$
\begin{aligned}
& R_{a b c d}=R_{a b c d}^{(p+1)}-K_{a c} K_{b d}+K_{b c} K_{a d}, \\
& R_{a n b c}=\nabla_{b} K_{a c}-\nabla_{c} K_{a b}, \\
& R_{a n b n}=R_{a b}-R_{a b}^{(p+1)}+K K_{a b}-K_{a c} K_{b}^{c},
\end{aligned}
$$

to evaluate

$$
\begin{aligned}
2 R_{\mathrm{lilj} j}= & -R_{i j}^{(p+1)}+R_{i j}+K K_{i j}-K_{i c} K_{j}^{c} \\
& +R_{T i T j}^{(p+1)}-K_{T T} K_{i j}+K_{i T} K_{T j}-\nabla_{i} K_{j T}-\nabla_{j} K_{i T}+2 \nabla_{T} K_{i j}=0
\end{aligned}
$$

The type I constraint (2.5) is written for the Weyl tensor components so generally we need to subtract the trace from the Riemann tensor expression (2.8). By construction our metrics solve Einstein equations so any traces of Riemann tensor vanish. However in order to avoid counting the same equations twice we can drop the Hamiltonian constraint (2.4) and use the type I constraint in the form (2.8). The equation (2.8) can be used to solve for $K_{i j}$ in terms of $K_{i T}$ with solution becoming much simpler for the large mean curvature hypersurfaces. The natural candidates for the large mean curvature hypersurfaces are black hole horizons.

\section{$3 \quad$ Kerr geometry}

The Kerr metric in Boyer-Lindquist (BL) coordinates is of the form

$d s^{2}=-\left(1-\frac{2 M r}{\rho^{2}}\right) d t^{2}-\frac{4 M a r \sin ^{2} \theta}{\rho^{2}} d t d \phi+\frac{\rho^{2}}{\Delta} d r^{2}+\rho^{2} d \theta^{2}+\left(r^{2}+a^{2}+\frac{2 M a^{2} r \sin ^{2} \theta}{\rho^{2}}\right) \sin ^{2} \theta d \phi^{2}$ $\rho^{2}=r^{2}+a^{2} \cos ^{2} \theta, \quad \Delta=r^{2}+a^{2}-2 M r$.

The Kerr metric is an algebraically special metric of type $\mathbf{D}$. The null frame in BL coordinates is given by the Kinnersley tetrad

$$
\begin{aligned}
\mathbf{k} & =\frac{r^{2}+a^{2}}{\Delta} \partial_{t}+\partial_{r}+\frac{a}{\Delta} \partial_{\phi}, \\
\mathbf{l} & =\frac{r^{2}+a^{2}}{2 \rho^{2}} \partial_{t}-\frac{\Delta}{2 \rho^{2}} \partial_{r}+\frac{a}{2 \rho^{2}} \partial_{\phi}, \\
\sqrt{2}(r+i a \cos \theta) m & =i a \sin \theta \partial_{t}+\partial_{\theta}+\frac{i}{\sin \theta} \partial_{\phi}, \\
\mathbf{k}^{\mu} \mathbf{l}_{\mu} & =-1=-m_{\mu} \bar{m}^{\mu} .
\end{aligned}
$$

For the type $\mathbf{D}$ metric the only nontrivial components of the Weyl tensor have boost weight zero and can be expressed via the complex $\Psi_{2}$ invariant $[14,15]$

$$
\Psi_{2} \equiv-C_{\mu \nu \lambda \rho} \mathbf{l}^{\mu} m^{\nu} \bar{m}^{\lambda} \mathbf{k}^{\rho}=\frac{M}{(r+i a \cos \theta)^{3}}
$$




\section{$3.1 \quad r=r_{0}=$ const surface}

A natural candidate for the fluid hypersurface is the $r=r_{0}=$ const hypersurface $\Sigma$ with the unit normal

$$
n^{\mu} \partial_{\mu}=\frac{\sqrt{\Delta}}{\rho} \partial_{r}
$$

The normal vector becomes null when $\Delta(r)=0$, what corresponds to the pair of $\mathrm{BH}$ horizons

$$
\Delta(r)=0=\left(r-r_{+}\right)\left(r-r_{-}\right), \quad r_{ \pm}=M \pm \sqrt{M^{2}-a^{2}} .
$$

The mean curvature of $\Sigma$

$$
K=h^{a b} K_{a b}=\nabla_{\mu} n^{\mu}=\frac{1}{\sqrt{|g|}} \partial_{\mu}\left(\sqrt{|g|} n^{\mu}\right)=\frac{\partial_{r} \Delta}{2 \sqrt{\Delta}}\left(r_{0}\right)+\frac{\partial_{r} \rho}{\rho} \sqrt{\Delta}\left(r_{0}\right)
$$

becomes large as we approach horizons. We can use $\Delta$ as small expansion parameter to describe the geometry of $\Sigma$ as it approaches the outer horizon $r=r_{+}$.

\subsection{Induced metric}

The induced metric on $\Sigma$ is

$d s^{2}=-\left(1-\frac{2 M r}{\rho^{2}}\right) d t^{2}-\frac{4 M a r \sin ^{2} \theta}{\rho^{2}} d t d \phi+\rho^{2} d \theta^{2}+\left(\frac{\left(r^{2}+a^{2}\right)^{2}-\Delta a^{2} \sin ^{2} \theta}{\rho^{2}}\right) \sin ^{2} \theta d \phi^{2}$,

with $r, \rho$ being evaluated at $r_{0}$. Fo the rest of the section we will discuss the hypersurface quantities, so in order to avoid an overusing the 0-subscript we will assume that $r=r_{0}$. The determinant of the induced metric

$$
\operatorname{det} h_{a b}=-\rho^{2} \Delta \sin ^{2} \theta,
$$

so the hypersurface indeed becomes null as $r_{0} \rightarrow r_{+}$. We can remove the $d t d \phi$ term by choosing the zero angular momentum observable (ZAMO) coordinates

$$
\phi \rightarrow \phi^{\prime}=\phi+\Omega_{0} t, \quad t \rightarrow t^{\prime}=t, \quad \Omega_{0}=\frac{2 M a r_{0}}{\left(a^{2}+r_{0}^{2}\right)^{2}-\Delta a^{2} \sin ^{2} \theta} .
$$

The metric (3.7) becomes

$d s^{2}=-\frac{\Delta \rho^{2} d t^{2}}{\left(r^{2}+a^{2}\right)^{2}-\Delta a^{2} \sin ^{2} \theta}+\rho^{2} d \theta^{2}+\left(\frac{\left(r^{2}+a^{2}\right)^{2}-\Delta a^{2} \sin ^{2} \theta}{\rho^{2}}\right) \sin ^{2} \theta\left(d \phi+t \partial_{\theta} \Omega_{0} d \theta\right)^{2}$.

In particular the leading $\Delta$-expansion

$$
\begin{aligned}
& h_{t t}=-\frac{\Delta \rho^{2}}{\left(r^{2}+a^{2}\right)^{2}}+\mathcal{O}\left(\Delta^{2}\right), \quad h_{t \phi}=h_{t \theta}=0, \quad h_{\phi \phi}=\frac{\left(r^{2}+a^{2}\right)^{2} \sin ^{2} \theta}{\rho^{2}}+\mathcal{O}(\Delta), \\
& h_{\phi \theta}=\frac{a^{3} \Delta t \sin 2 \theta \sin ^{2} \theta}{\rho^{2}\left(r^{2}+a^{2}\right)}+\mathcal{O}\left(\Delta^{2}\right), \quad h_{\theta \theta}=\rho^{2}+\mathcal{O}\left(\Delta^{2}\right) .
\end{aligned}
$$




\subsection{Extrinsic curvature}

The extrinsic curvature on the hypersurface is defined via the Lie derivative

$$
\mathcal{L}_{n} g_{\mu \nu}=n^{\sigma} \partial_{\sigma} g_{\mu \nu}+\partial_{\mu} n^{\sigma} g_{\sigma \nu}+\partial_{\nu} n^{\sigma} g_{\mu \sigma}
$$

Since our normal vector has only $r$-component and the Kerr metric (3.1) has $g_{r t}=g_{r \phi}=$ $g_{r \theta}=0$ the Lie derivative simplifies into

$$
2 K_{a b} d x^{a} d x^{b}=\left.\mathcal{L}_{n} d s^{2}\right|_{\Sigma}=\left.n^{\mu} \partial_{\mu} d s^{2}\right|_{\Sigma}=\frac{\sqrt{\Delta}}{\rho} \partial_{r} d s^{2} .
$$

For our hypersurface after the coordinate transform (3.9) we have the following extrinsic curvature components

$$
\begin{aligned}
K_{t t} & =-\frac{\rho \partial_{r} \Delta \sqrt{\Delta}}{2\left(r^{2}+a^{2}\right)^{2}}+\mathcal{O}\left(\Delta^{3 / 2}\right), \quad K_{\theta \phi}=\mathcal{O}\left(\Delta^{3 / 2}\right), \quad K_{t \theta}=\mathcal{O}\left(\Delta^{3 / 2}\right), \\
K_{t \phi} & =r a \sqrt{\Delta} \frac{\sin ^{2} \theta}{\rho^{3}}\left[1+\frac{\rho^{2} \partial_{r} \Delta}{2 r\left(a^{2}+r^{2}\right)}\right]+\mathcal{O}\left(\Delta^{3 / 2}\right), \quad K_{\theta \theta}=\frac{r \sqrt{\Delta}}{\rho}+\mathcal{O}\left(\Delta^{3 / 2}\right), \\
K_{\phi \phi} & =\frac{\sqrt{\Delta} \sin ^{2} \theta}{2 \rho^{5}}\left(2 r\left(r^{2}+a^{2}\right)\left[2 \rho^{2}-\left(r^{2}+a^{2}\right)\right]-a^{2} \rho^{2} \partial_{r} \Delta \sin ^{2} \theta\right)+\mathcal{O}\left(\Delta^{3 / 2}\right) .
\end{aligned}
$$

\subsection{Null frame}

The fluid/gravity correspondence require algebraic speciality with respect to the null frame constructed from the hypersurface data $n, T$. In practice we often have a null frame of maximal speciality written for the full bulk metric. Given a null frame we can construct the unit vector

$$
n=\frac{\mathbf{k}-\mathbf{l}}{\sqrt{2}},
$$

but it can fail to be hypersurface orthogonal, i.e. obey

$$
n_{[\mu} \partial_{\nu} n_{\lambda]}=0
$$

Moreover, we need $\Sigma$ to have large mean curvature $K=\nabla_{\mu} n^{\mu}$, so there is no canonical fluid hypersurface for a generic algebraically special geometry. Fortunately for the Kerr geometry we have a family of $r=$ const surfaces that approach the horizon and serve as natural candidates for $\Sigma$. For these hypersurface the mean curvature (3.6) becomes large as we approach the horizon, but they do not preserve the full type $\mathbf{D}$ speciality. Let us figure out how much speciality can we preserve on $\Sigma$.

The null frame (3.2) on $\Sigma$ changes under the coordinate transformation (3.9) to become

$$
\begin{aligned}
\mathbf{k} \rightarrow \mathbf{k} & =\frac{r^{2}+a^{2}}{\Delta} \partial_{t}+\partial_{r}-\frac{a \rho^{2}}{\left(a^{2}+r^{2}\right)^{2}-\Delta a^{2} \sin ^{2} \theta} \partial_{\phi}, \\
\mathbf{l} \rightarrow \mathbf{l} & =\frac{r^{2}+a^{2}}{2 \rho^{2}} \partial_{t}-\frac{\Delta}{2 \rho^{2}} \partial_{r}-\frac{1}{2} \frac{\Delta}{\left(a^{2}+r^{2}\right)^{2}-\Delta a^{2} \sin ^{2} \theta} \partial_{\phi} .
\end{aligned}
$$


Moreover we can further rescale vectors $\mathbf{k} \rightarrow \frac{\sqrt{\Delta}}{\sqrt{2} \rho} \mathbf{k}$ and $\mathbf{l} \rightarrow \frac{\sqrt{2} \rho}{\sqrt{\Delta}} \mathbf{l}$ so that

$$
\begin{aligned}
\mathbf{k} & =\frac{T+n}{\sqrt{2}}-\frac{a \sqrt{\Delta} \rho}{\sqrt{2}\left(r^{2}+a^{2}\right)^{2}} \partial_{\phi}+\mathcal{O}\left(\Delta^{3 / 2}\right) \partial_{\phi}+\mathcal{O}(\Delta) T, \\
\mathbf{l} & =\frac{T-n}{\sqrt{2}}-\frac{a \sqrt{\Delta} \rho}{\sqrt{2}\left(r^{2}+a^{2}\right)^{2}} \partial_{\phi}+\mathcal{O}\left(\Delta^{3 / 2}\right) \partial_{\phi}+\mathcal{O}(\Delta) T,
\end{aligned}
$$

with unit normal to $\Sigma$ and the time-foliation normal for the induced metric (3.10) on $\Sigma$ being

$$
n=\frac{\sqrt{\Delta}}{\rho} \partial_{r}, \quad T=\frac{\sqrt{\left(r^{2}+a^{2}\right)^{2}-\Delta a^{2} \sin ^{2} \theta}}{\rho \sqrt{\Delta}} \partial_{t}=\frac{r^{2}+a^{2}}{\rho \sqrt{\Delta}} \partial_{t}+\mathcal{O}(\sqrt{\Delta}) .
$$

The difference between the $\frac{T \pm n}{\sqrt{2}}$ null frame and the canonical type $\mathbf{D}$ null frame (3.18) can be schematically written as $\sqrt{\Delta} f^{i} m_{i}$. The possible corrections to the algebraic constraints from the different weight components have the following schematic form

$$
\begin{aligned}
& \delta C_{\mathbf{k} i \mathbf{k} j} \sim \delta C_{\mathbf{l i l j} j}=2 \sqrt{\Delta} f^{k} C_{\mathbf{l} i k j}+\Delta f^{k} f^{l} C_{k i l j}=\mathcal{O}(\Delta), \\
& \delta C_{\mathbf{k} i j k} \sim \delta C_{\mathbf{l} i j k}=\sqrt{\Delta} f^{l} C_{l i j k}=\mathcal{O}(\sqrt{\Delta}) .
\end{aligned}
$$

In the second equality we used the type $\mathbf{D}$ constraints and the near-horizon scaling of the boost weight zero components (3.3). Using (2.7) we can estimate the leading order expressions for the Weyl tensor components in terms of the hypersurface data to be

$$
C_{\mathbf{l i l j} j} \sim C_{\mathbf{k} i \mathbf{k} j}=\mathcal{O}\left(\Delta^{0}\right), \quad C_{\mathbf{l} i j k} \sim C_{\mathbf{k} i j k}=\mathcal{O}(\sqrt{\Delta}) .
$$

Thus the corrections (3.20) does not affect only the leading order for the type $\mathbf{I}_{i}$ constraints. So effectively we can say that the choice of $\Sigma$ breaks the Kerr geometry speciality from the type $\mathbf{D}$ to the subtype $\mathbf{I}_{i}$, which has

$$
C_{\mathrm{lil} j}=C_{\mathbf{k} i \mathbf{k} j}=0
$$

\section{Slowly rotating black hole}

We can consider a simplified version of the Kerr geometry (3.1) with $a / M \ll 1$, so we can drop the higher orders in $a$. Such toy version contains almost all features of the full Kerr geometry while being very easy to analyze.

\subsection{Geometry}

The Kerr metric (3.1) at leading order in $a$ becomes

$d s^{2}=-f^{2} d t^{2}-\frac{4 M a \sin ^{2} \theta}{r} d t d \phi+f^{-2} d r^{2}+r^{2}\left(d \theta^{2}+\sin ^{2} \theta d \phi^{2}\right)+\mathcal{O}\left(a^{2}\right), \quad f^{2}=1-\frac{2 M}{r}$. 
The $r=r_{0}$ hypersurface has the following geometric data

$$
\begin{aligned}
n & =f_{0} \partial_{r}, \quad T=f_{0}^{-1} \partial_{t}, \\
d s^{2} & =-f_{0}^{2} d t^{2}-\frac{4 M a \sin ^{2} \theta}{r_{0}} d t d \phi+r_{0}^{2}\left(d \theta^{2}+\sin ^{2} \theta d \phi^{2}\right)+\mathcal{O}\left(a^{2}\right), \\
K_{a b} d x^{a} d x^{b} & =-f_{0}^{2} \partial_{r} f_{0} d t^{2}+\frac{2 M a f_{0} \sin ^{2} \theta}{r_{0}^{2}} d t d \phi+r_{0} f_{0}\left(d \theta^{2}+\sin ^{2} \theta d \phi^{2}\right)+\mathcal{O}\left(a^{2}\right) .
\end{aligned}
$$

Using the coordinate transformation

$$
\phi \rightarrow \phi+\frac{2 M a}{r_{0}^{3}} t
$$

we can remove the $d t d \phi$ term, so the geometry simplifies into

$$
\begin{aligned}
d s^{2} & =-f_{0}^{2} d t^{2}+r_{0}^{2}\left(d \theta^{2}+\sin ^{2} \theta d \phi^{2}\right)+\mathcal{O}\left(a^{2}\right) \\
K_{a b} d x^{a} d x^{b} & =-f_{0}^{2} \partial_{r} f_{0} d t^{2}+\frac{6 M a f_{0} \sin ^{2} \theta}{r_{0}^{2}} d t d \phi+r_{0} f_{0}\left(d \theta^{2}+\sin ^{2} \theta d \phi^{2}\right)+\mathcal{O}\left(a^{2}\right) .
\end{aligned}
$$

The mean curvature

$$
K=K_{a b} h^{a b}=\partial_{r} f_{0}+\frac{2 f_{0}}{r_{0}}=\frac{M}{f_{0} r_{0}^{2}}+\frac{2 f_{0}}{r_{0}}
$$

becomes large as we approach the black hole horizon at $r=2 M$ since $r_{0} f_{0}^{2}=\left(r_{0}-2 M\right)$. Moreover the leading near-horizon behavior of the mean curvature is related to the Hawking temperature $T_{H}$ for the slowly rotating Kerr solution.

$$
2 \pi T_{H}=\left.\frac{-K_{t t}}{\sqrt{-g_{t t}}}\right|_{r_{0}=r_{H}=2 M}=\left.f_{0} \partial_{r} f_{0}\right|_{r_{0}=r_{H}=2 M}=\frac{M}{r_{H}^{2}}=\frac{1}{4 M}
$$

The type $\mathbf{D}$ null frame (3.2) after the coordinate transformation (4.3) becomes

$$
\begin{aligned}
\mathbf{k} & =f_{0}^{-2}\left(\partial_{t}-\frac{2 M a}{r_{0}^{3}} \partial_{\phi}\right)+\partial_{r}+\frac{a}{r_{0}^{2} f_{0}^{2}} \partial_{\phi}+\mathcal{O}\left(a^{2}\right), \\
\mathbf{l} & =\frac{1}{2}\left(\partial_{t}-\frac{2 M a}{r_{0}^{3}} \partial_{\phi}\right)-\frac{f_{0}^{2}}{2} \partial_{r}+\frac{a}{2 r_{0}^{2}} \partial_{\phi}+\mathcal{O}\left(a^{2}\right),
\end{aligned}
$$

We can freely rescale our null vectors $\mathbf{k} \rightarrow f_{0} \mathbf{k} / \sqrt{2}, \quad \mathbf{l} \rightarrow \sqrt{2} \mathbf{l} / f_{0}$ without changing the null frame

$$
\begin{aligned}
\mathbf{k} & =\frac{1}{f_{0} \sqrt{2}} \partial_{t}+\frac{f_{0}}{\sqrt{2}} \partial_{r}+\frac{a f_{0}}{\sqrt{2} r_{0}^{2}} \partial_{\phi}+\mathcal{O}\left(a^{2}\right)=\frac{1}{\sqrt{2}}(T+n)+\frac{a f_{0}}{\sqrt{2} r_{0}^{2}} \partial_{\phi}+\mathcal{O}\left(a^{2}\right) \\
\mathbf{l} & =\frac{1}{f_{0} \sqrt{2}} \partial_{t}-\frac{f_{0}}{\sqrt{2}} \partial_{r}+\frac{a f_{0}}{\sqrt{2} r_{0}^{2}} \partial_{\phi}+\mathcal{O}\left(a^{2}\right)=\frac{1}{\sqrt{2}}(T-n)+\frac{a f_{0}}{\sqrt{2} r_{0}^{2}} \partial_{\phi}+\mathcal{O}\left(a^{2}\right) .
\end{aligned}
$$

to arrive into canonical form (2.6), used for the dual fluid construction. The additional contribution along the $\partial_{\phi}$ breaks the type $\mathbf{D}$ to type $\mathbf{I}_{i}$ (3.22). The first relation in (3.22) is the type $\mathbf{I}$ relation which we can use to describe the dual fluid. The second relation in (3.22) imposes additional relations for the dual fluid, which we will describe in fluid variables later. 


\subsection{Dual fluid}

Our geometry (4.4) can be written in terms of $\tau=\lambda^{-2} t$ and $\lambda^{2}=f_{0}^{2}=\mathcal{O}\left(r_{0}-2 M\right)$

$$
d s^{2}=-\lambda^{-2} d \tau^{2}+\gamma_{i j}(x) d x^{i} d x^{j}, \quad K=\mathcal{O}\left(\lambda^{-1}\right),
$$

to simplify the large mean curvature expansion analysis. The type $\mathbf{I}$ constraint (2.8) for metric (4.9) simplifies into

$2 C_{\mathbf{l i l j}}=-\lambda D_{i} K_{j \tau}-\lambda D_{j} K_{i \tau}+2 \lambda \partial_{\tau} K_{i j}-R_{i j}^{(p)}+\left(\gamma^{k l} K_{k l}-2 \lambda^{2} K_{t t}\right) K_{i j}-K_{i k} K_{j}^{k}+2 \lambda^{2} K_{i \tau} K_{j \tau}$

with $R_{i j}^{(p)}, D_{i}$ being Ricci tensor and covariant derivative with respect to the spatial metric $\gamma_{i j}$. The large mean curvature $K=\mathcal{O}\left(\lambda^{-1}\right)$ motivates to search for a perturbative in $\lambda$ solution to the type I constraint (4.10) using

$$
\lambda K_{i \tau}=\sum_{k=0}^{\infty} \lambda^{k} K_{i \tau}^{(k)}, \quad K_{i j}=\sum_{k=1}^{\infty} \lambda^{k} K_{i j}^{(k)}, \quad \lambda^{3} K_{\tau \tau}=\sum_{k=0}^{\infty} \lambda^{k} K_{\tau \tau}^{(k)} .
$$

The solution for $K_{i j}$ is

$$
2 K_{\tau \tau}^{(0)} K_{i j}^{(1)}=-D_{i} K_{j \tau}^{(0)}-D_{j} K_{i \tau}^{(0)}-R_{i j}^{(p)}+2 K_{i \tau}^{(0)} K_{j \tau}^{(0)} .
$$

The covariant conservation equation (2.3) for our geometry (4.9) becomes

$$
\begin{aligned}
D^{i} K_{i \tau}-\gamma^{i j} \partial_{\tau} K_{i j} & =0 \\
-\lambda^{2} \partial_{\tau} K_{i \tau}+D^{j} K_{i j}-\partial_{i}\left(\gamma^{k l} K_{k l}\right)+\lambda^{2} \partial_{i} K_{\tau \tau} & =0 .
\end{aligned}
$$

The equation (4.14) at leading orders $\mathcal{O}\left(\lambda^{-1}, \lambda^{0}\right)$ order is

$$
\partial_{i} K_{\tau \tau}^{(0)}=0, \quad \partial_{i} K_{\tau \tau}^{(1)}=0 .
$$

which is the statement that the $\mathrm{BH}$ temperature (4.6) is the same at each point on the horizon. The next order equation is

$$
-\partial_{\tau} K_{i \tau}^{(0)}+D^{j} K_{i j}^{(1)}-\partial_{i}\left(\gamma^{k l} K_{k l}^{(1)}\right)+\partial_{i} K_{\tau \tau}^{(1)}=0 .
$$

Let us define the fluid velocity $v_{i}$

$$
v_{i}(x, \tau) \equiv K_{i \tau}^{(0)}
$$

viscosity $\eta$ and pressure $p$

$$
\eta^{-1}(x, \tau) \equiv-K_{\tau \tau}^{(0)}, \quad \eta>0, \quad p(x, \tau) \equiv-K_{\tau \tau}^{(2)} .
$$

The solution (4.12) in the new notation is of the form

$$
2 K_{i j}^{(1)}=-2 \eta v_{i} v_{j}+\eta\left(D_{i} v_{j}+D_{j} v_{i}\right)+\rho R_{i j}^{(p)} .
$$


The time component of the constraint equation (4.13) becomes the incompressibility condition for the dual fluid

$$
D_{i} v^{i}=0
$$

while the other component (4.16)

$$
\partial_{\tau} v^{i}+\eta v_{j} D^{j} v^{i}-\frac{1}{2} \eta D_{j}\left(D^{i} v^{j}+D^{j} v^{i}\right)+\partial^{i}\left[p+\frac{1}{4} \eta R^{(p)}-\eta v^{2}\right]=0,
$$

becomes the Navier-Stokes equation in curved space

$$
\partial_{\tau} v^{i}+v_{j} D^{j} v^{i}-\frac{1}{2} \eta D_{j}\left(D^{i} v^{j}+D^{j} v^{i}\right)+\partial^{i} p=0
$$

if we redefine pressure and velocity

$$
v^{i} \rightarrow \eta^{-1} v_{i}, \quad \eta p+\frac{1}{4} \eta^{2} R^{(p)}-v^{2} \rightarrow p .
$$

The equation (4.22) describes the shear perturbation for the Schwarzschild metric [12], so it is not surprising that it describes the dual fluid for the slowly-rotating Kerr black hole.

\subsection{Type $\mathbf{I}_{i}$ fluid as a Killing flow}

Our geometry (4.1) and the fluid hypersurface admits an additional vanishing Weyl tensor component (3.22). We already have the same number of fluid variables and fluid equations so any additional constraints would restrict the generality of the fluid solution. The additional constraint can be written

$$
0=C_{\mathbf{k} i \mathbf{k} j}-C_{\mathbf{l i l j}}=C_{n i T j}+C_{T i n j}=R_{n i T j}+R_{T i n j}-\frac{2}{p} \gamma_{i j} R_{n T},
$$

so we can use the Riemann tensor instead. The leading order expression in fluid variables

$$
R_{n i T j}+R_{\text {Tinj }}=0 \Rightarrow D_{i} v_{j}+D_{j} v_{i}=\mathcal{L}_{v} \gamma_{i j}=0 .
$$

The equation (4.25) is a Killing equation, so the solution for a fluid velocity can be written as

$$
v_{i}(x, \tau)=f(\tau) k_{i}(x)
$$

with $k_{i}(x)$ being a Killing vector for the spatial metric $\gamma_{i j}$. The substitution of (4.26) into (4.22) lead to the equation

$$
\partial_{\tau} v^{i}=\partial^{i}\left(\frac{1}{2} v^{2}-p\right) .
$$

We can integrate over the fluid on compact spatial section of the horizon

$$
\partial_{\tau} \int \sqrt{\gamma} v^{2}=\partial_{\tau}\left(f^{2}\right) \int k^{2} \sqrt{\gamma}=0
$$

to conclude that $\partial_{\tau} f=0$. So we can summarize that Killing-based solution (4.26) solves the NS equation (4.22) with constant $f(\tau)$, that is imposed by compactness of the Kerr horizon. The solution is

$$
v_{i}=k_{i}(x), \quad P=\frac{1}{2} k_{i} k^{i}(x)+\text { const. }
$$


The solution (4.29) is generic for any type $\mathbf{I}_{i}$ metric with a hypersurface geometry (4.9) and large mean curvature. Our slowly rotating Kerr geometry (4.4) obeys these properties so we can compare extrinsic curvature from (4.4) and the NS solution (4.29). The dual fluid velocity

$$
v^{i} \partial_{i}=\eta^{-1} \gamma^{i j} K_{\tau j} \partial_{i}=\frac{3 a}{r_{0}^{2}} \partial_{\phi}
$$

is $\tau$-independent Killing vector on the round two sphere. Indeed our result for the hypersurface geometry (4.9) match the generic solution for toy example (4.29).

Our toy example illustrates an feature, that are common for all type $\mathbf{D}$ dual fluids: fluid velocity is closely related to the Killing vectors of the metric. The NS equation in curved space (4.22) always admits solutions from Killing vectors (4.29). On the gravity side it is conjectured that the type $\mathbf{D}$ metrics have some number of Killing vectors and fluid/gravity mapping lead to Killing equation as one of the additional constraints present for type $\mathbf{D}$ metrics.

\section{Dual fluid for rotating black hole}

The most studied fluid system is the incompressible Navier-Stokes (NS) equation, so we want to use additional physical assumptions to write our fluid system in the form similar to the NS equation. In particular we will use the near-horizon expansion with small parameter $\lambda$ to control the relative size of various terms and simplify our equations.

\subsection{Geometry}

The induced metric on the hypersurface is of the form (3.11)

$$
d s^{2}=h_{a b} d x^{a} d x^{b}=-\lambda^{2} h(x) d t^{2}+\gamma_{i j}\left(x, \lambda^{2} t\right) d x^{i} d x^{j} .
$$

where we identify $\lambda^{2} r^{2}=\Delta$. The smallness $h_{t t}=\mathcal{O}\left(\lambda^{2}\right)$ is a generic feature when $\Sigma$ approaches the black hole horizon and becomes null. The second property, $\partial_{t} \gamma_{i j}=\mathcal{O}\left(\lambda^{2}\right)$, is related to the fact that all point on the black hole horizon move with the same angular velocity $\Omega_{H}$. Let us do a time redefinition $\tau=\lambda^{2} t$ so the metric takes the form

$$
d s^{2}=-h(x) \frac{d \tau^{2}}{\lambda^{2}}+\gamma_{i j}(x, \tau) d x^{i} d x^{j}, \quad \partial_{\tau} \gamma_{i j}=\mathcal{O}\left(\lambda^{0}\right) .
$$

We chose $\tau=$ const as a time foliation so the unit normal is of the form

$$
T=\lambda h^{-1 / 2} \partial_{\tau}
$$

Using our explicit metric ansatz (5.2) we can rewrite the type I constraint (2.8)

$$
\begin{aligned}
2 C_{\mathrm{lil} j}= & -\lambda h^{-1} D_{i}\left(h^{1 / 2} K_{j \tau}\right)-\lambda h^{-1} D_{j}\left(h^{1 / 2} K_{i \tau}\right)+2 \lambda h^{-1 / 2} \partial_{\tau} K_{i j}+h^{-3 / 2} \lambda^{3} \partial_{\tau} \gamma_{i j} K_{\tau \tau} \\
& -\frac{1}{2} \lambda h^{-1 / 2} \partial_{\tau} \gamma_{i l} \gamma^{k l} K_{k j}-\frac{1}{2} \lambda h^{-1 / 2} \partial_{\tau} \gamma_{j l} \gamma^{k l} K_{i k}+D_{i} D_{j} \log h+\frac{1}{2} \partial_{i} \log h \partial_{j} \log h \\
& -R_{i j}^{(p)}+\left(K-\lambda^{2} h^{-1} K_{\tau \tau}\right) K_{i j}-K_{i k} K_{j}^{k}+2 \lambda^{2} h^{-1} K_{i \tau} K_{j \tau}+\mathcal{O}\left(\lambda^{2}\right) .
\end{aligned}
$$




\subsection{Near-horizon expansion}

Using the large mean curvature $K=\mathcal{O}\left(\lambda^{-1}\right)$ of a hypersurface and the $\lambda$-expansion

$$
\lambda K_{i \tau}=\sum_{k=0}^{\infty} \lambda^{k} K_{i \tau}^{(k)}, \quad K_{i j}=\sum_{k=1}^{\infty} \lambda^{k} K_{i j}^{(k)}, \quad \lambda^{3} K_{\tau \tau}=\sum_{k=0}^{\infty} \lambda^{k} K_{\tau \tau}^{(k)},
$$

we can solve for $K_{i j}$

$$
\begin{aligned}
2 K_{\tau \tau}^{(0)} K_{i j}^{(1)}= & -D_{i}\left(h^{1 / 2} K_{j \tau}^{(0)}\right)-D_{j}\left(h^{1 / 2} K_{i \tau}^{(0)}\right)+h^{-1 / 2} \partial_{\tau} \gamma_{i j} K_{\tau \tau}^{(0)}+ \\
& h D_{i} D_{j} \log h+\frac{1}{2} h \partial_{i} \log h \partial_{j} \log h-h R_{i j}^{(p)}+2 K_{i \tau}^{(0)} K_{j \tau}^{(0)}
\end{aligned}
$$

The covariant conservation (2.3) for our metric becomes

$$
2 h^{-1} \lambda D^{i}\left(h^{1 / 2} K_{i \tau}\right)-h^{-3 / 2} \lambda^{3} \gamma^{i j} \partial_{\tau} \gamma_{i j} K_{\tau \tau}-2 \lambda h^{-1 / 2} \gamma^{i j} \partial_{\tau} K_{i j}+\lambda h^{-1 / 2} K^{i j} \partial_{\tau} \gamma_{i j}=0
$$

and

$-\lambda^{2} \partial_{\tau}\left(h^{-1 / 2} K_{\tau i}\right)+D^{j}\left(\sqrt{h} K_{i j}\right)-\sqrt{h} \partial_{i}\left(\gamma^{k l} K_{k l}\right)-\frac{1}{2} h^{-1 / 2} \lambda^{2} \partial_{\tau} \log \gamma K_{\tau i}+\lambda^{-2} \partial_{i}\left(h^{-1 / 2} K_{\tau \tau}\right)=0$.

Our equation at leading $\mathcal{O}\left(\lambda^{-1}, \lambda^{0}\right)$ orders is

$$
\partial^{i}\left(h^{-1 / 2} K_{\tau \tau}^{(0)}\right)=0, \quad \partial^{i}\left(h^{-1 / 2} K_{\tau \tau}^{(1)}\right)=0,
$$

which is the statement that the $\mathrm{BH}$ temperature is the same at each point on the horizon. The next order is

$-\partial_{\tau}\left(h^{-1 / 2} K_{\tau i}^{(0)}\right)+D^{j}\left(\sqrt{h} K_{i j}^{(1)}\right)-\sqrt{h} \partial_{i}\left(\gamma^{k l} K_{k l}^{(1)}\right)-\frac{1}{2} h^{-1 / 2} \partial_{\tau} \log \gamma K_{\tau i}^{(0)}+\partial_{i}\left(h^{-1 / 2} K_{\tau \tau}^{(2)}\right)=0$.

Similarly to the slow rotating case let us introduce the fluid velocity $v_{i}$ as

$$
v_{i}(x, \tau) \equiv h^{1 / 2} K_{i \tau}^{(0)},
$$

viscosity $\rho$ and pressure $p$

$$
\eta^{-1}(x, \tau) \equiv-h^{-1 / 2} K_{\tau \tau}^{(0)}, \quad p(x, \tau) \equiv-h^{-1 / 2} K_{\tau \tau}^{(2)} .
$$

The solution (5.6) in the new notation is of the form

$2 \sqrt{h} K_{i j}^{(1)}=-2 \eta h^{-1} v_{i} v_{j}+\eta\left(D_{i} v_{j}+D_{j} v_{i}\right)-\eta h D_{i} D_{j} \log h-\frac{1}{2} \eta h \partial_{i} \log h \partial_{j} \log h+\eta h R_{i j}^{(p)}+\partial_{\tau} \gamma_{i j}$.

The time-component of the constraint equations (5.7) becomes the incompressibility equation

$$
\eta D_{i} v^{i}+\frac{1}{2} \partial_{t} \log \gamma=0,
$$


while the spatial component (5.10) becomes the NS-like equation

$$
\begin{aligned}
h^{-1} \partial_{\tau} v_{i}+\eta h^{-1} v^{j} D_{j} v_{i}-\eta h^{-2} v_{i} v_{j} \partial^{j} h-\frac{1}{2} \eta D^{j}\left(D_{i} v_{j}+D_{j} v_{i}+\eta^{-1} \partial_{\tau} \gamma_{i j}\right)+\frac{1}{2} \eta h^{-2} v^{2} \partial_{i} h \\
+\partial_{i}\left(p+\frac{1}{4} \eta h R^{(p+1)}+\frac{1}{4} \eta D^{2} h-\eta h^{-1} v^{2}\right)=0 .
\end{aligned}
$$

The equation (5.15) is not the NS equation in curved space, since there are several additional terms. So, unfortunately, the fluid gravity map for the Kerr black hole cannot be used to generate a new solution to the usual NS system. However, we should notice that the (5.15) depends on the coordinate choice for the hypersurface metric (5.2). For example we can choose the Gaussian normal coordinates, where $h(x)=1$ at the price of more complicated $\tau$-dependence for the spatial metric $\gamma_{i j}$. Thus, the reasonable questions about the dual fluid should be formulated in invariant form. In the remaining part of the paper we will describe two features of the dual fluid (Killing-based solution and linearized stability), that are independent on particular coordinate choice and are also present for the usual NS equation.

\subsection{The type $I_{i}$ fluid as a Killing flow}

The additional constraint for type $\mathbf{I}_{i}$ geometries $C_{\mathbf{k} i \mathbf{k} j}=0$ can be written using Riemann tensor

$$
R_{n i T j}+R_{T i n j}=0 \Rightarrow \Sigma_{i k} \equiv D_{i} v_{j}+D_{j} v_{i}+\eta^{-1} \partial_{\tau} \gamma_{i j}=0 .
$$

The NS equation (5.15) can be rewritten using $\Sigma_{i j}$

$h^{-1} \gamma_{i j} \partial_{\tau} v^{j}+\eta h^{-1} v^{j} \Sigma_{i j}-\eta h^{-2} v_{i} v_{j} \partial^{j} h-\frac{1}{2} \eta D^{j} \Sigma_{i j}+\partial_{i}\left(p+\frac{1}{4} \eta h R^{(p+1)}+\frac{1}{4} \eta D^{2} h-\frac{3}{2} \eta h^{-1} v^{2}\right)=0$,

so the additional constraint (5.16) reduces equation into

$$
h^{-1} \dot{v}^{i}-\eta h^{-2} v^{i} v^{k} \partial_{k} h+\partial^{i}\left(p+\frac{1}{4} \eta h R^{(p+1)}+\frac{1}{4} \eta D^{2} h-\frac{3}{2} \eta h^{-1} v^{2}\right)=0 .
$$

Furthermore, algebraically special solutions of type $\mathbf{D}$ conjectured to have some number of Killing vectors [10]. The additional constraint (5.16) can be written as a Killing equation

$$
\partial_{\tau} \gamma_{i j}=D_{j} w_{i}+D_{i} w_{j}, \quad D_{i} v_{j}+D_{j} v_{i}+\eta^{-1} \partial_{\tau} \gamma_{i j}=D_{i}\left(v_{j}+\eta^{-1} w_{j}\right)+D_{j}\left(v_{i}+\eta^{-1} w_{i}\right),
$$

since for Kerr geometry and some other examples time dependence of the metric $\gamma$ comes from a coordinate transformation $x^{i} \rightarrow x^{i}+w^{i} \tau$ (3.9). In case of Kerr geometry both fluid velocity $v_{i}$ and $w_{i}$ are aligned with $\partial_{\phi}$ bulk Killing vector so $v^{k} \partial_{k} h$ term vanishes. In particular the fluid velocity for our explicit Kerr data:

$$
v^{i} \partial_{i}=h^{1 / 2} \gamma^{i j} K_{\tau j}^{(0)} \partial_{i}=\frac{r^{3} a}{\left(r^{2}+a^{2}\right)^{3}}\left[1+\frac{\rho^{2} \partial_{r} \Delta}{2 r\left(a^{2}+r^{2}\right)}\right] \partial_{\phi} .
$$

Thus the NS equation for the Kerr case is solved by the static velocity, that is almost Killing vector. Given existence of the multiple Killing vectors for a higher dimensional type $\mathbf{D}$ geometries we can conjecture that the corresponding dual fluid solutions have similar structure of the static almost Killing flows. 


\section{Stability, Reynolds number, turbulence}

The most interesting phenomenon accompanying the fluid dynamics is the turbulence. So it might be very fruitful to see what can we say about it using the fluid/gravity correspondence. There are two major directions of turbulence studying: developing turbulence and developed turbulence. The first direction address questions about instabilities of the fluid flow, their emergence, dynamics and interactions. So in context of developing turbulence we are interesting in a transition process from smooth flow to a chaotic one. In case of developed turbulence we usually assume that the flow is sufficiently chaotic, so we can study interesting relations for the statistically averaged quantities. We do not have much to say about the developed turbulence while we can use our explicit results for the Kerr fluid to study its stability properties.

Let us use our toy example to describe the notion of stability and its features. The NS equation on static curved background is

$$
\partial_{\tau} v_{i}+v^{j} D_{j} v_{i}-\eta D^{j}\left(D_{i} v_{j}+D_{j} v_{i}\right)+\partial_{i} p=0, \quad D_{i} v^{i}=0 .
$$

For a given solution $V, P$ we can consider a small perturbation on top of it

$$
v \rightarrow V+v, \quad p \rightarrow P+p,
$$

that satisfies the following linearized equation

$$
\partial_{\tau} v_{i}+V^{j} D_{j} v_{i}+v^{j} D_{j} V_{i}-\eta D^{j}\left(D_{i} v_{j}+D_{j} v_{i}\right)+\partial_{i} p=0, \quad D_{i} v^{i}=0 .
$$

Our equations (6.3) are linear so it is useful to expand $v(x, \tau)$ in Fourier modes. For a given mode $\hat{v}(\omega, k)$ its time evolution $e^{-i \omega \tau}$ is governed by the frequency $\omega$. In general $\omega$ is a complex number, so given a positive imaginary part we may have a perturbations that grow with time. It is natural do define solution $V, P$ to the NS system (6.1) as a stable solution if the linearized equation (6.3) has no Fourier modes with negative imaginary part, i.e. all linearized solutions decay with time. A complete analysis for linearized stability using mode expansions can only be done for simplest fluid solutions, so we need use some other methods for generic flows.

The powerful approach to linearized stability is based on energy balance equation[16]. Let us take the linearized equation (6.3), multiply it by $v_{i}$ and integrate over the time slice of the horizon

$$
\frac{1}{2} \partial_{\tau} \int \sqrt{\gamma} v^{2}=-\frac{\eta}{2} \int \sqrt{\gamma}\left(D_{j} v_{i}+D_{i} v_{j}\right)^{2}-\int \sqrt{\gamma} v^{i} v^{k} D_{k} V_{i} .
$$

The lefthandside measures time dependence for the kinetic energy of the fluid perturbation while the rightahndside describes the energy dissipation by viscosity term and energy flow from background solution. The relative size for the two terms in (6.4) can be described in terms of dimensionless combination

$$
R e=\frac{L v}{\eta}
$$


so that stability requires

$$
R e<R e_{c} .
$$

The critical value is determined by minimization

$$
\operatorname{Re}_{c} \equiv \min \frac{\frac{1}{2} \int \sqrt{\gamma}\left(D_{j} v_{i}+D_{i} v_{j}\right)^{2}}{-\int \sqrt{\gamma} v^{i} v^{k} D_{k} V_{i}}
$$

over divergence free velocities. This bound was first proposed by O.Reynolds [17]. Existence of the minimum is guarantied because of the same (quadratic) velocity dependence for both functionals. Moreover, the contribution to energy balance from the quadratic term, that we dropped in linearized analysis, is a total derivative

$$
v^{i} v^{j} D_{j} v_{i}=\frac{1}{2} D_{j}\left(v^{j} v^{2}\right)
$$

So this extra term does not affect our analysis. Let us apply the energy balance analysis to the toy solution and to generic rotational black hole solution.

Reynolds number for the toy solution (4.30)

$$
R e=\frac{v L}{\eta}=3 \frac{a}{M}=\frac{3 J}{M^{2}} .
$$

By construction it is parametrically small for slow-rotating Kerr, what is usually sufficient for stable fluid. Nevertheless let us evaluate the $R e_{c}$. Our toy case fluid solution (4.29) has trivial energy flow from background solution

$$
\int \sqrt{\gamma} v^{i} v^{k} D_{k} V_{i}=\frac{1}{2} \int \sqrt{\gamma} v^{i} v^{k}\left(D_{k} V_{i}+D_{i} V_{k}\right)=0
$$

since background fluid velocity obeys Killing equation. So the Killing flow solution (4.29) for slowly rotating black hole has $R e_{c}=\infty$ and it is always stable.

For the generic Kerr we have the following linearized system

$$
D_{i} v^{i}=0
$$

$$
\begin{array}{r}
h^{-1} \gamma_{i j} \partial_{\tau} v^{j}+\eta h^{-1} v^{j} \Sigma_{i j}+\eta h^{-1} V^{j}\left(D_{i} v_{j}+D_{j} v_{i}\right)-\eta h^{-2} V_{i} v_{j} \partial^{j} h-\eta h^{-2} v_{i} V_{j} \partial^{j} h \\
-\frac{1}{2} \eta D^{j}\left(D_{i} v_{j}+D_{j} v_{i}\right)+\partial_{i} p=0
\end{array}
$$

The extra terms are similar to the ones described in [13] and describe various physical effects like Coriolis force.

The energy balance equation is

$\gamma^{-1 / 2} h^{-1} \partial_{\tau}\left(\gamma^{1 / 2} v^{2}\right)=-\frac{1}{2} \eta\left(D_{i} v_{j}+D_{j} v_{i}\right)^{2}-\eta h^{-1} v^{i} v^{j} \Sigma_{i j}+\eta h^{-2} v^{2} V_{j} \partial^{j} h-D_{i}\left(v^{i} p+\ldots\right)$.

The type $\mathbf{I}_{i}$ condition $\Sigma_{i j}=0$ and bulk Killing symmetry $V^{k} \partial_{k} h=0$ simplify energy balance into

$$
\partial_{\tau} \int h^{-1} v^{2} \sqrt{\gamma}=-\frac{1}{2} \eta \int\left(D_{i} v_{j}+D_{j} v_{i}\right)^{2} \sqrt{\gamma}
$$

what makes the fluid, dual to rotating black hole, stable. 


\section{$7 \quad$ Summary of the results}

We described the generalization of the algebraically special fluid/gravity correspondence, which includes rotating black holes in four and higher dimensions. On a gravity side rotating black holes are type $\mathbf{D}$ algebraically special while the duality construction requires to choose the null frame with smaller speciality. The reduced speciality is of type $\mathbf{I}_{i}$, which is still bigger then the minimal type $\mathbf{I}$ required by the duality. Thus the dual fluid obeys additional equations, which are very similar to the Killing equations. In case of slowlyrotating Kerr fluid velocity is the Killing vector for the $S^{2}$, which is the constant time slice of the horizon. For a generic Kerr solution fluid velocity is closely related to the bulk Killing vector and this pattern seems to have a generalization to higher dimensions.

The dual fluid system for slowly-rotating Kerr is a simple generalization of the incompressible Navier-Stokes equation to the curved background. For a generic Kerr solution the Navier-Stokes equation is modified by additional terms. The structure of additional terms depends on a particular choice of the hypersurface metric. Interestingly, the modified Navier-Stokes equation admits a Killing-like solution, similarly to the incompressible fluid on a sphere. The energy balance equation for the Killing-flow background indicates that the solution is stable.

Based on our analysis it is reasonable to conjecture that the dual fluid to a generic type $\mathbf{D}$ geometry is Killing-like flow, that obeys the modified Navier-Stokes equation. Such flows seem to be stable according to energy balance equation, so there no instabilities and turbulence. This is unfortunate as type $\mathbf{D}$ geometries are most studied solutions to Einstein equations in four and higher dimensions, since they have a big number of Killing vectors. So, in order to describe a flow, that has unstable regime and investigate gravity-dual of turbulence we might need to consider less special algebraic types such as types $\mathbf{I I}$ or $\mathbf{I}_{i}$. Fortunately for us some number of such solutions is known so it might very fruitful to construct their fluid duals.

\section{Acknowledgments}

Author is grateful to A. Strominger for useful discussions on early stages of this work. This work was supported in part by DOE grant DE-SC0011632 and the Sherman Fairchild scholarship.

Open Access. This article is distributed under the terms of the Creative Commons Attribution License (CC-BY 4.0), which permits any use, distribution and reproduction in any medium, provided the original author(s) and source are credited.

\section{References}

[1] R.H. Price and K.S. Thorne, Membrane Viewpoint on Black Holes: Properties and Evolution of the Stretched Horizon, Phys. Rev. D 33 (1986) 915 [INSPIRE].

[2] T. Damour and M. Lilley, String theory, gravity and experiment, Les Houches 87 (2008) 371 [arXiv: 0802.4169] [INSPIRE]. 
[3] S. Bhattacharyya, V.E. Hubeny, S. Minwalla and M. Rangamani, Nonlinear Fluid Dynamics from Gravity, JHEP 02 (2008) 045 [arXiv:0712.2456] [INSPIRE].

[4] S. Bhattacharyya, S. Minwalla and S.R. Wadia, The Incompressible Non-Relativistic Navier-Stokes Equation from Gravity, JHEP 08 (2009) 059 [arXiv:0810.1545] [INSPIRE].

[5] I. Bredberg, C. Keeler, V. Lysov and A. Strominger, Wilsonian Approach to Fluid/Gravity Duality, JHEP 03 (2011) 141 [arXiv: 1006.1902] [INSPIRE].

[6] I. Bredberg, C. Keeler, V. Lysov and A. Strominger, From Navier-Stokes To Einstein, JHEP 07 (2012) 146 [arXiv:1101.2451] [INSPIRE].

[7] D.T. Son and A.O. Starinets, Viscosity, Black Holes and Quantum Field Theory, Ann. Rev. Nucl. Part. Sci. 57 (2007) 95 [arXiv: 0704.0240] [INSPIRE].

[8] R. Baier, P. Romatschke, D.T. Son, A.O. Starinets and M.A. Stephanov, Relativistic viscous hydrodynamics, conformal invariance and holography, JHEP 04 (2008) 100 [arXiv: 0712.2451] [INSPIRE].

[9] V. Lysov and A. Strominger, From Petrov-Einstein to Navier-Stokes, arXiv:1104.5502 [INSPIRE].

[10] A. Coley, Classification of the Weyl Tensor in Higher Dimensions and Applications, Class. Quant. Grav. 25 (2008) 033001 [arXiv:0710.1598] [INSPIRE].

[11] S. Bhattacharyya, S. Lahiri, R. Loganayagam and S. Minwalla, Large rotating AdS black holes from fluid mechanics, JHEP 09 (2008) 054 [arXiv:0708.1770] [INSPIRE].

[12] I. Bredberg and A. Strominger, Black Holes as Incompressible Fluids on the Sphere, JHEP 05 (2012) 043 [arXiv: 1106.3084] [inSPIRE].

[13] C.-J. Chou, X. Wu, Y. Yang and P.-H. Yuan, Rotating Black Holes and Coriolis Effect, Phys. Lett. B 761 (2016) 131 [arXiv:1511.08691] [INSPIRE].

[14] A. Petrov, Einstein Spaces, Pergamon Press (1969).

[15] E. Hertl, C. Hoenselaers, D. Kramer, M. Maccallum and H. Stephani, Exact solutions of Einstein's field equations, Cambridge University Press (2003).

[16] L. Landau and E. Lifshitz, Fluid Mechanics, volume 6 of Course of Theoretical Physics, first edition, Pergamon Press (1975).

[17] O. Reynolds, On the dynamical theory of incompressible viscous fluids and the determination of the criterion, Phil. Trans. Roy. Soc. London 186 (1894) 123. 